\title{
TITLE:
}

\section{Volume transition of nematic gels in nematogenic solvents}

$\operatorname{AUTHOR}(S)$ :

Urayama, K; Okuno, Y; Nakao, T; Kohjiya, S

CITATION:

Urayama, K ...[et al]. Volume transition of nematic gels in nematogenic solvents. JOURNAL OF CHEMICAL PHYSICS 2003, 118(6): 2903-2910

ISSUE DATE:

2003-02-08

URL:

http://hdl.handle.net/2433/39748

RIGHT:

Copyright 2003 American Institute of Physics. This article may be downloaded for personal use only. Any other use requires prior permission of the author and the American Institute of Physics. 


\title{
Volume transition of nematic gels in nematogenic solvents
}

\author{
Kenji Urayama, ${ }^{\text {a) }}$ Yuko Okuno, Toshio Nakao, and Shinzo Kohjiya \\ Institute for Chemical Research, Kyoto University, Uji, Kyoto-fu 611-0011, Japan
}

(Received 24 July 2002; accepted 13 November 2002)

Equilibrium swelling and phase behavior of liquid crystalline (LC) networks swollen in miscible nematogenic solvents has been investigated by polarizing microscopy as a function of temperature. Four systems, i.e., each of two different LC networks comprising dissimilar mesogens in two different nematic solvents, exhibit essentially the same swelling and phase characteristics. The swelling characteristics strongly correlate with the phases of the LC molecules inside and outside the gel. The two independent nematic-isotropic transition temperatures for the gel $\left(T_{N I}^{G}\right)$ and the surrounding pure solvent $\left(T_{N I}^{S} ; T_{N I}^{G}>T_{N I}^{S}\right.$ for all the systems examined) yield three characteristic temperature regions. In the totally isotropic and nematic phases ( $T>T_{N I}^{G}$ and $T<T_{N I}^{S}$, respectively), the degree of equilibrium swelling $(Q)$ is almost independent of $T$, and the magnitudes of $Q$ in these phases are comparable. Meanwhile, $Q$ strongly depends on $T$ in the region $T_{N I}^{S}<T<T_{N I}^{G}$ where the LC phases inside and outside the gel are different, i.e., nematic and isotropic, respectively. Upon cooling down to $T_{N I}^{G}$ from the totally isotropic phase, the nematic network and the miscible nematic solvent inside the gel form a single nematic phase, which causes a discontinuous decrease in gel volume: The swollen isotropic gel is discontinuously transformed into the shrunken nematic gel. As temperature decreases further, $Q$ of the shrunken nematic gel surrounded by the isotropic LC solvent increases again, and $Q$ at $T \approx T_{N I}^{S}$ reaches almost the same magnitude as that in the totally isotropic phase. The nematic ordering of the surrounding pure solvent takes place at $T_{N I}^{S}$, which yields an inflection of the $Q-T$ curve without discontinuity. A mean field theory for nematic gel successfully describes the swelling and phase behavior observed. The theory also demonstrates that the nematic-isotropic transition of gel drives a volume transition; an increase in nematic order inside gel induces a further swelling of nematic gel surrounded by isotropic LC solvent. (C) 2003 American Institute of Physics. [DOI: 10.1063/1.1535896]

\section{INTRODUCTION}

Volume phase transition phenomena of polymer gels have been extensively investigated over the last 20 years. It has been reported that an infinitesimal change in an environmental intensive variable such as solvent composition, ${ }^{1-3}$ $p \mathrm{H},{ }^{4,5}$ and temperature ${ }^{6-10}$ yields a discontinuous volume change for some polymer gels. These volume transitions are driven by the balance between the repulsive and attractive forces acting on the network chains such as van der Waals, hydrophobic, ionic, hydrogen bonding. ${ }^{7,11}$ In previous communication, ${ }^{12}$ we reported the first observation of the volume transition for liquid crystalline (LC) gels in a nematic solvent at the nematic-isotropic transition temperature of the gels $\left(T_{N I}^{G}\right)$. This volume transition is triggered by nematic ordering of LC molecules inside the gel. This finding adds a new driving molecular force for volume transition phenomena. "Temperature-sensitive gels" exhibiting volume transition at a certain temperature have attracted much attention of scientist and technologists because of their applications to drug delivery systems and sensors, etc. ${ }^{13-15}$ So far $N$-isopropylacrylamide-based gels have been known as a typical temperature-sensitive gel. LC gels undergoing a sharp and large volume change accompanied by nematic-isotropic

\footnotetext{
a) Author to whom all correspondence should be addressed. Electronic mail: urayama@scl.kyoto-u.ac.jp
}

transition at $T_{N I}^{G}$ is a new type of temperature-sensitive gel, which will extend the potential application of polymer gels to industrial device. LC networks swollen in LC solvent have also been expected as a soft actuator driven under electric field utilizing the anisotropic character that the orientation of mesogen is controllable by external field. ${ }^{16-18}$ Elucidation of the correlation between swelling and phase behavior will provide the basis to develop further the applications of LC gels.

In a previous communication, ${ }^{12}$ we also reported that the shrunken namatic gel swelled again with decreasing in temperature in the region $T_{N I}^{S}<T<T_{N I}^{G}$ where $T_{N I}^{S}$ is the nematic-isotropic transition temperature of the surrounding pure LC solvent. The interesting swelling characteristics such as volume transition and reswelling behavior were observed in the nematic networks swollen in a dissimilar nematogen. In the present study, the swelling behavior of four systems, i.e., each of two different LC networks comprising dissimilar mesogens in two different nematic solvents, is investigated to confirm the universality of the nematic ordering induced-volume transition as well as the reentrant swelling behavior. A nematic network swollen in a similar nematogen is also examined to clarify if the dissimilarity between mesogen on network and nematic solvent is responsible for the characteristic swelling behavior. It is also interesting to survey how $T_{N I}^{G}$ of swollen LC network depends on each of 
transition temperatures of solvent-free (dry) LC network $\left(T_{N I}^{G}\right)$ and nematic solvent $\left(T_{N I}^{S}\right): T_{N I}^{G}$ is much different from $T_{N I}^{G *}$ due to the presence of a miscible nematic solvent inside the gel. The data of $T_{N I}$ for the four systems will provide the basis to discuss this issue. Of further interest is that a mean-field theory ${ }^{19-21}$ for nematic gel where the nematic ordering effect is considered on the basis of the MaierSaupe concept predicts the volume transition as well as the reswelling behavior observed experimentally. The temperature dependence of the degree of equilibrium swelling in each system is quantitatively compared with the theoretical prediction.

\section{MEAN FIELD THEORY FOR NEMATIC GEL IN NEMATIC SOLVENT}

We describe here an outline of a mean field theory ${ }^{19-21}$ for equilibrium swelling and phase behavior of a nematic gel in a nematic solvent. The mean field theory is originally for main-chain LC networks, but it may also be applicable to side-chain LC networks although the dangling mesogenbackbone polymer interaction and the effect of totally nonmesomorphic backbone on solubility are not explicitly introduced. ${ }^{19-24}$ Chiu et al. ${ }^{23,24}$ showed that the experimental phase diagrams for the mixtures of side-chain LC polymers and low molar mass nematic LC were well described by the mean field approach. They considered that the dangling mesogen-backbone polymer interaction had already been reflected in the nematic-isotropic transition temperature; the effect of nonmesomorphic backbone on solubility was reflected in the Flory-Huggins mixing interaction parameter. ${ }^{23,24}$ As will be demonstrated later, along the same line, the mean field theory for nematic gel in nematic solvent also successfully describes the swelling and phase behavior of the side-chain LC networks in the LC solvents observed experimentally.

Consider a nematic gel composed of long flexible nonmesomorphic components (spacers) and rigid mesogens. The repeating unit on a network chain between adjacent crosslinks consists of a mesogen occupying the number $n_{m}$ of sites (segments) and a spacer occupying the number $n_{s}$ of sites. The quantity $n_{m}$ corresponds to the axial ratio of the mesogen. The total number of segments on a network chain $n$ is given by $n=\left(n_{m}+n_{s}\right) t$ where $t$ is the number of a repeating unit. The degree of equilibrium swelling $Q$ is expressed as

$$
Q=\frac{V}{V_{0}}=\frac{a^{3} N_{t}}{a^{3} n N_{g}}=\frac{1}{\phi},
$$

where $V=a^{3} N_{t}$ and $V_{0}$ are the volumes of the network in the swollen and dry state, respectively, $a^{3}$ is the volume of an unit segment, and $\phi$ is the volume fraction of the network. The total number of unit cells $N_{t}$ inside the gel is given by $N_{t}=n N_{g}+n_{0} N_{0}$ where $N_{g}$ and $N_{0}$ are the numbers of the network chains and solvents inside the gel, respectively, and $n_{0}$ is the axial ratio of the nematic solvent, i.e., the number of sites occupied by one nematic solvent molecule.
The total free energy of the nematic gel can be expressed as a sum of free energy of isotropic mixing $F_{\text {mix }}$, free energy of nematic ordering $F_{\text {nem }}$, free energy due to elasticity of nematic network $F_{e l}$, as follows:

$$
F=F_{\text {mix }}+F_{\text {nem }}+F_{e l} \text {. }
$$

The free energy of isotropic mixing $F_{\text {mix }}$ may be described in terms of the Flory-Huggins theory: ${ }^{25}$

$$
\begin{aligned}
\frac{F_{m i x}}{N_{t} k T}= & \frac{(1-\phi)}{n_{0}} \ln (1-\phi)+\chi_{m s} \phi_{m} \phi_{s}+\chi_{m 0} \phi_{m}(1-\phi) \\
& +\chi_{s 0} \phi_{s}(1-\phi),
\end{aligned}
$$

where $k$ is the Boltzmann constant, $T$ is the absolute temperature, $\phi_{m}=a^{3} n_{m} t N_{g} / V=(1-p) \phi$ and $\phi_{s}=p \phi$ are the volume fractions of mesogen and spacer, respectively, and $p$ $=n_{s} /\left(n_{m}+n_{s}\right)$ is the fraction of spacer segment. The quantities $\chi_{m s}, \chi_{m 0}$ and $\chi_{s 0}$ are the Flory-Huggins isotropic mixing interaction parameters for mesogen-spacer segments, mesogen-solvent molecules, and spacer-solvent molecules, respectively. We assume hereafter $\chi=\chi_{m s}=\chi_{s 0}$ and $\chi_{m 0}=0$. The latter means that the solvent acts as an athermal solvent for the mesogen.

The free energy of nematic ordering $F_{\text {nem }}$ is given by the Maier-Saupe mean field theory ${ }^{26,27}$ and its generalization to binary nematogens mixtures ${ }^{23,24,28,29}$ as follows:

$$
\begin{aligned}
\frac{F_{\mathrm{nem}}}{N_{t} k T}= & \sum_{i=m, 0} \frac{\phi_{i}}{n_{i}} f\left(\theta_{i}\right) \ln 4 \pi f\left(\theta_{i}\right) d \Omega_{i}-\frac{1}{2} \nu_{m m} \phi_{m}^{2} S_{m}^{2} \\
& -\frac{1}{2} \nu_{00}(1-\phi)^{2} S_{0}^{2}-\nu_{m 0} \phi_{m}(1-\phi) S_{m} S_{0},
\end{aligned}
$$

where $\Omega_{i}$ denotes the solid angle, $\theta_{i}(i=m, 0)$ is the angle between a reference axis and the director of each liquid crystal molecule. The subscripts $m$ and 0 refer to the mesogen and the solvent inside the gel, respectively. The orientational order parameter of each nematogen $S_{i}(i=m, 0)$ is defined as

$$
S_{i}=\int\left(\frac{3}{2} \cos ^{2} \theta_{i}-\frac{1}{2}\right) f\left(\theta_{i}\right) d \Omega_{i} .
$$

The function $f\left(\theta_{i}\right)(i=m, 0)$ is the normalized orientation distribution function which may be expressed by

$$
f\left(\theta_{i}\right)=\frac{1}{Z_{i}} \exp \left[\eta_{i}\left(\frac{3}{2} \cos ^{2} \theta_{i}-\frac{1}{2}\right)\right],
$$

where $Z_{i}(i=m, 0)$ is the partition function defined as $Z_{i}$ $=\int \exp \left[\eta_{i}\left(3 \cos ^{2} \theta_{i}-1\right) / 2\right] d \Omega_{i}$, and $\eta_{i}(i=m, 0)$ is a dimensionless measure representing the strength of the nematic field. The quantity $\nu_{m m}$ is the Maier-Saupe nematic interaction parameter between the mesogens, $\nu_{00}$ is that between the solvent, and $\nu_{m 0}$ is that between the mesogen and the solvent. The nematic-isotropic transition temperature of the pure solvent $T_{N I}^{S}$ is related to $\nu_{00}$ via inverse dependence on $T$ as $^{27}$

$$
\nu_{00}=\frac{4.54}{n_{0}}\left(\frac{T_{N I}^{S}}{T}\right) .
$$

In the simulations of $T$ dependence of $Q$ in Ref. 20 and 21, $\nu_{m m}=\nu_{m 0}=\nu_{00}$ was assumed for simplicity even when me- 
sogen of gel and nematic solvent were dissimilar. In the present study, it was needed to distinguish $\nu_{i j}(i, j=m, 0)$ to obtain the satisfactory data-fit. The cross-interaction $\nu_{m 0}$ is not known, thus we assume that $\nu_{m 0}$ is proportional to $\left(\nu_{m m} \nu_{00}\right)^{1 / 2}$ with a proportionality constant $\kappa$ different from unity as ${ }^{23,29}$

$$
\nu_{m 0}=\kappa\left(\nu_{m m} \nu_{00}\right)^{1 / 2} .
$$

The constant $\kappa$ characterizes the relative strength of the cross-interaction between the two dissimilar nematogens as compared to that in the same nematogens. The case $\kappa=1$ shows the intermediate coupling where the cross-interaction in the mixture has the same magnitude as that between the same nematogens in their pure states. The elastic free energy $F_{e l}$ for nematic network can be derived from the classical rubber elasticity theory combined with the freely jointed rod model as a function of $\phi$ and orientational order parameter of the mesogen $S_{m}:$ :

$$
\begin{aligned}
\frac{F_{e l}}{N_{t} k T}= & \frac{3}{2 n}\left\{\left[\frac{\phi}{n\left(1+2 S_{m}\right)\left(1-S_{m}\right)^{2}}\right]^{1 / 3}+\frac{\phi}{3} \ln \left(1+2 S_{m}\right)\right. \\
& \left.\times\left(1-S_{m}\right)^{2}-\phi\left(1-\frac{2}{3} \ln \sqrt{n} \phi\right)\right\}
\end{aligned}
$$

When $S_{m}=0$, Eq. (9) accords with the elastic free energy of isotropic network. Warner et al. ${ }^{19,22}$ derived another expression of $F_{e l}$ for nematic network. It is shown ${ }^{31}$ that their expression has essentially the same form of Eq. (9) in the dilute systems $(\phi \ll 1)$; the use of their expression instead of Eq. (9) for $F_{e l}$ yields qualitatively the same results.

The relations between $\eta_{m}, \eta_{0}, S_{m}$ and $S_{0}$ are evaluated by minimization of the free energy [Eq. (2)] with respect to $S_{m}$ and $S_{0}$ :

$$
\begin{aligned}
\eta_{m}= & n_{m}\left[\nu_{m m} \phi_{m} S_{m}+\nu_{m 0}(1-\phi) S_{0}\right] \\
& -\frac{3 n_{m} S_{m}}{n \phi_{m}\left(1+2 S_{m}\right)\left(1-S_{m}\right)} \\
& \times\left\{\left[\frac{\phi}{n\left(1+2 S_{m}\right)\left(1-S_{m}\right)^{2}}\right]^{1 / 3}-\phi\right\}
\end{aligned}
$$

and

$$
\eta_{0}=n_{0}\left[\nu_{m 0} \phi_{m} S_{m}+\nu_{00}(1-\phi) S_{0}\right] .
$$

The order parameter of the pure solvent outside the gel $\left(S_{b}\right)$ is given by Eqs. (5) and (6) $(i=b)$ with

$$
\eta_{b}=n_{0} \nu_{00} S_{b} .
$$

Swelling equilibrium requires that the chemical potentials of the solvents inside and outside the gel $\left(\mu_{0}\right.$ and $\mu_{0}^{\circ}$, respectively) are equivalent:

$$
\mu_{0}\left(\phi, S_{m}, S_{0}\right)=\mu_{0}^{\circ}\left(S_{b}\right) .
$$

Thus the equilibrium condition is obtained from Eq. (13) as follows:

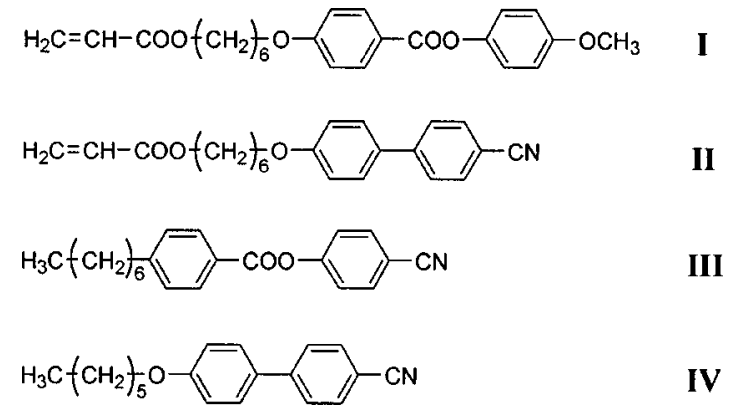

FIG. 1. Molecular structures of the employed liquid crystalline monomers and solvents.

$$
\begin{aligned}
& \frac{1}{n}\left\{\left[\frac{\phi}{n\left(1+2 S_{m}\right)\left(1-S_{m}\right)^{2}}\right]^{1 / 3}-\phi\right\}+\frac{1}{n_{0}} \ln (1-\phi)+\frac{\phi}{n_{0}} \\
& \quad-\chi_{m s} p \phi^{2}(1-p)+\chi_{m 0}\left(1-p \phi^{2}\right)+\chi_{s 0} p \phi^{2} \\
& \quad+\frac{1}{2} \nu_{m m} S_{m}^{2} \phi_{m}^{2}+\nu_{m 0} S_{m} S_{0} \phi_{m}(1-\phi) \\
& \quad+\frac{1}{2} \nu_{00}\left[S_{0}^{2}(1-\phi)^{2}-S_{b}^{2}\right]+\frac{1}{n_{0}} \ln \frac{Z_{b}}{Z_{0}}=0 .
\end{aligned}
$$

The equilibrium values of $Q(=1 / \phi), S_{m}, S_{0}$, and $S_{b}$ as a function of $T$ are determined by solving Eqs. (5) (for $i$ $=m, 0, b)$ and (14) self-consistently.

\section{EXPERIMENT}

\section{A. Sample preparation}

Two side chain LC networks (designated as LCN-I and LCN-II) were prepared from the mesogenic acrylate monomers I and II (Fig. 1), respectively. The details of the preparation of these networks were described in our previous paper. $^{12}$ Molar ratio of monomer I or II, crosslinker $(1,6-$ Hexanediol diacrylate) and initiator $\left(2,2^{\prime}\right.$-azobisiso butyronitrile) was 98:1:1. The cylindrical gels with the diameters of several hundreds micron were made by radical polymerization in capillaries. After the reaction, the gels were immersed in toluene to remove fully unreacted molecules. The gels were then gradually deswollen in mixtures of toluene and methanol by increasing the methanol content stepwise. The fully deswollen gels were completely dried in air. The dried gels were allowed to swell in a nematic LC solvent (molecule III or IV in Fig. 1). The nematic LC III with a quoted purity of at least 99\% was purchased from Aldrich $\mathrm{Co}$, and it was used as received without further purification. The nematic LC IV, synthesized by essentially the same method as in the case of the monomer II, was employed after purification. Nematic-isotropic transition temperatures $T_{N I}$ of the dry LC networks and the LC solvents III and IV, which were estimated in cooling process by polarizing optical microscopy, are listed in Table I.

\section{B. Swelling measurement and phase observation}

Measurement of gel diameters and the phase observation were made as a function of temperature by a Nikon polarizing optical microscope E600 POL equipped with a Linkam 
TABLE I. Nematic-isotropic transition temperatures of the liquid crystalline networks in the dry and swollen states and volume fractions of network in the vicinity of transition temperature.

\begin{tabular}{lcccccc}
\hline \hline & & \multicolumn{2}{c}{ in LC III $\left(T_{N I}^{S}=54.2^{\circ} \mathrm{C}\right)$} & & \multicolumn{2}{c}{ in LC IV $\left(T_{N I}^{S}=74.9^{\circ} \mathrm{C}\right)$} \\
\cline { 3 - 4 } \cline { 6 - 7 } Network sample & $T_{N I}^{G}{ }_{*}$ a $/{ }^{\circ} \mathrm{C}$ & $\phi_{N I}{ }^{\mathrm{b}}$ & $T_{N I}^{G} /{ }^{\circ} \mathrm{C}$ & & $\phi_{N I}{ }^{\mathrm{b}}$ & $T_{N I}^{G} /{ }^{\circ} \mathrm{C}$ \\
\hline LCN-I & 125.4 & 0.0740 & $58.2(59.5)^{\mathrm{c}}$ & & 0.0534 & $77.7(77.6)^{\mathrm{c}}$ \\
LCN-II & 131.2 & 0.183 & $71.0(68.3)^{\mathrm{c}}$ & & 0.131 & $79.6(82.3)^{\mathrm{c}}$ \\
\hline \hline
\end{tabular}

${ }^{\mathrm{a}}$ In the dry state.

${ }^{\mathrm{b}}$ Volume fraction of network in the swollen phase at $T=T_{N I}^{G}+0.2{ }^{\circ} \mathrm{C}$.

${ }^{\mathrm{c} C a l c u l a t e d}$ by Eq. (15).

LK-600PM sample stage under a nitrogen atmosphere. The details of the measurements were described elsewhere. ${ }^{12}$ The surface level of LC solvent in an optical cell was adjusted to be low enough so that the LC phases inside the gels as well as the boundary of the gel surface were distinctly visible when viewed through the microscope (but high enough to immerse the gel completely). Degree of equilibrium swelling $(Q)$ at each temperature was determined by the ratio of the gel diameters in the dry and swollen states $\left(d_{0}\right.$ and $d_{s}$, respectively) using the definition of $Q$ for isotropic swelling: $Q=V / V_{0}=\left(d_{s} / d_{0}\right)^{3}$ where $V$ and $V_{0}$ are the gel volumes in the swollen and dry states, respectively. The gels exhibited isotropic swelling even in the nematic phase because of the polydomain structure where the orientation of directors is globally random in space but a high orientational order is locally maintained.

\section{RESULTS}

Part (a) of Figs. 2-5 shows the degree of equilibrium swelling $(Q)$ as a function of temperature $(T)$ for each of LCN-I and LCN-II swollen in the LC solvent III or IV (designated as LCN-I/III, LCN-I/IV, LCN-II/III, and LCN-II/IV, respectively). The data were obtained in cooling process. There exists the two independent nematic-isotropic transition temperatures $T_{N I}^{G}$ and $T_{N I}^{S}$ for the gel and the surrounding pure solvent, respectively. For all the systems examined here, $T_{N I}^{G}$ is higher than $T_{N I}^{S}$. In the range $T<T_{N I}^{G}$, the nematic network and the miscible nematic solvent inside the gel form a single nematic phase. Phase separation inside the gel was not observed by polarizing microscopy with a typical magnification used in the studies ${ }^{32,33}$ on phase separation of LC mixtures. As can be seen in Table I, the four systems show different $T_{N I}^{G}$ depending on the network and the solvent used; $T_{N I}^{G}$ of each swollen LC network is much depressed relative to $T_{N I}^{G}$ in the dry state due to the presence of a large amount of the miscible nematic solvent whose $T_{N I}^{S}$ is fairly lower than $T_{N I}^{G_{*}}$.

According to the LC phases inside and outside the gel, there exists three characteristic temperature regions: $T>T_{N I}^{G}$ where the whole system is isotropic; $T_{N I}^{S}<T<T_{N I}^{G}$ where the phase inside the gel is nematic, while the surrounding pure LC solvent is isotropic; $T<T_{N I}^{S}$ where the system is totally nematic. As is evident from part (a) of Figs. 2-5, the swelling behavior is closely related to the phase characteristics; all the systems examined show essentially the same correlation between the swelling and phase behavior. The descriptions below are common to all the systems. In the totally isotropic phase $\left(T>T_{N I}^{G}\right), Q$ is almost independent of $T$, although $Q$ slightly decreases with decreasing in $T$ for LCN-I/III and LCN-II/III. At $T_{N I}^{G}$, the isotropic gel discontinuously shrinks into the nematic gel: A discontinuous large decrease in gel volume, i.e., volume transition, takes place as a result of
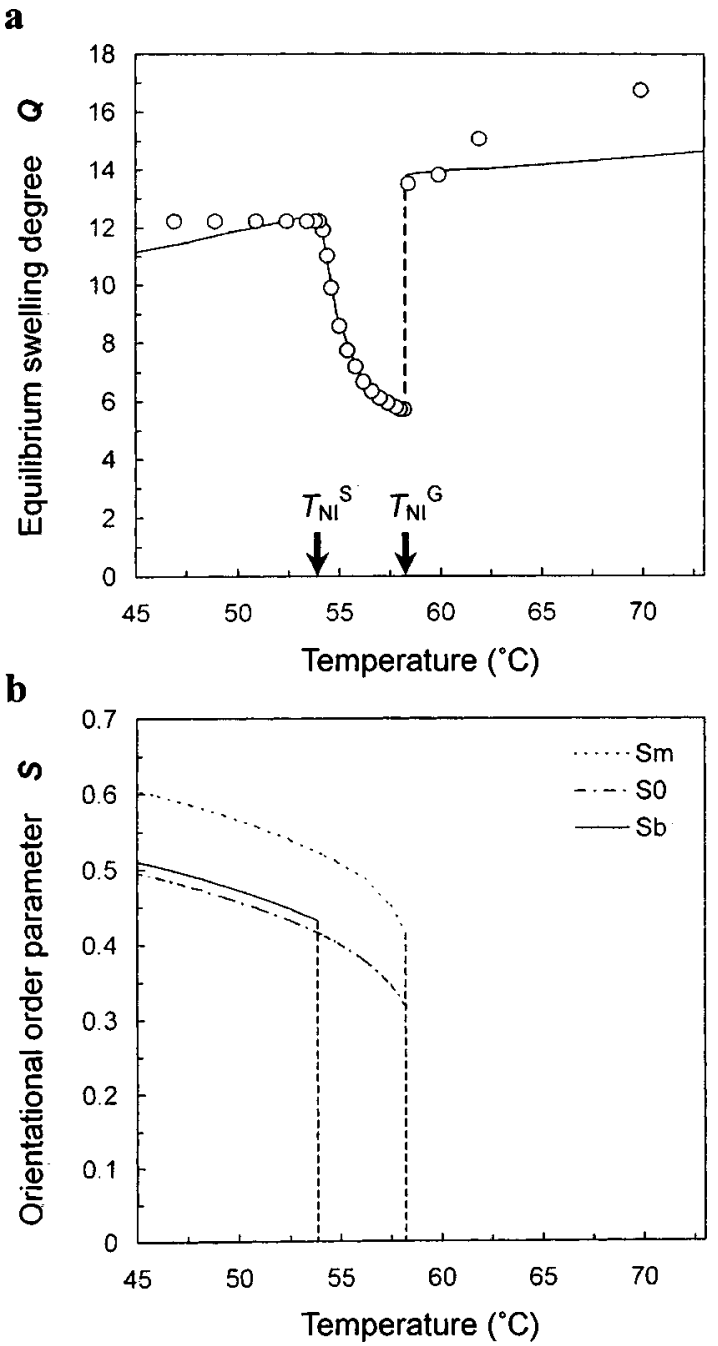

FIG. 2. (a) Equilibrium swelling degree $(Q)$ of LCN-I in the LC solvent III as a function of $T$. The nematic-isotropic transition temperature of the gel $\left(T_{N I}^{G}\right)$ and the surrounding pure LC solvent $\left(T_{N I}^{S}\right)$ are 58.2 and $54.2^{\circ} \mathrm{C}$, respectively. The solid line represent the theoretical swelling curve calculated with $n=120, n_{m}=3.3, p=0.23, n_{0}=2.5, \nu_{m m} / \nu_{00}=1.05, \kappa=0.966$, $\nu_{00} / \chi=0.5$. (b) The orientational order parameters of the mesogen on network $\left(S_{m}\right)$, the nematic solvents inside and outside gel $\left(S_{0}\right.$ and $S_{b}$, respectively) as a function of $T$ calculated using the same parameter values as (a). 
$\mathbf{a}$

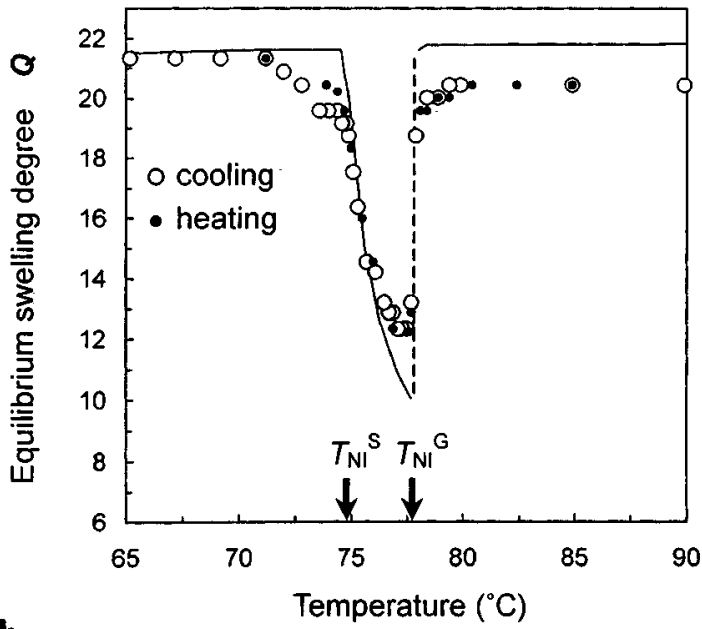

b

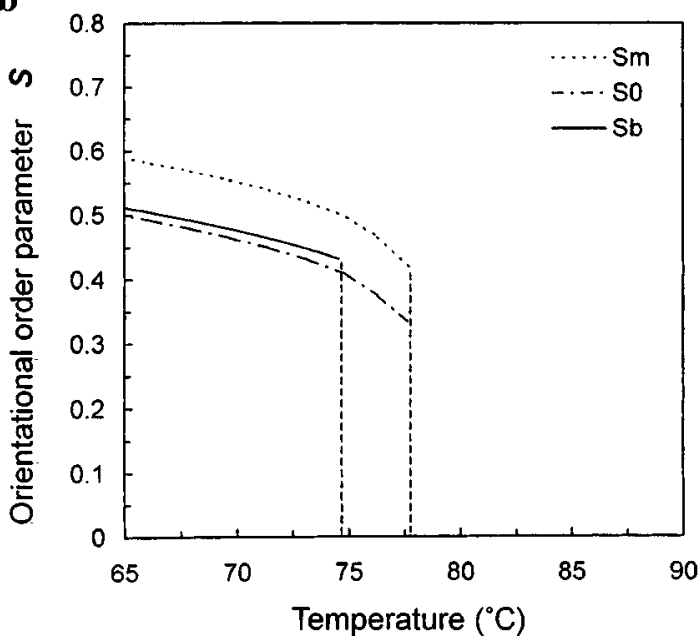

FIG. 3. (a) Equilibrium swelling degree $(Q)$ of LCN-I in the LC solvent IV as a function of $T$. The nematic-isotropic transition temperature of the gel $\left(T_{N I}^{G}\right)$ and the surrounding pure LC solvent $\left(T_{N I}^{S}\right)$ are 77.7 and $74.9^{\circ} \mathrm{C}$, respectively. The solid line represent the theoretical swelling curve calculated with $n=120, n_{m}=3.3, p=0.23, n_{0}=2.55, \nu_{m m} / \nu_{00}=1.0, \kappa=0.985$, $\nu_{00} / \chi=1.0$. (b) The orientational order parameters of the mesogen on network $\left(S_{m}\right)$, the nematic solvents inside and outside gel $\left(S_{0}\right.$ and $S_{b}$, respectively) as a function of $T$ calculated using the same parameter values as (a).

nematic ordering inside the gel. The volume transition and the nematic-isotropic transition of the gel take place simultaneously and sharply within $0.2^{\circ} \mathrm{C}$. As temperature decreases further, $Q$ of the shrunken nematic gel surrounded by isotropic LC solvent increases again. When temperature decreases down to $T \approx T_{N I}^{S}, Q$ of the nematic gel reaches almost the same magnitude as $Q$ in the totally isotropic phase $\left(T>T_{N I}^{G}\right)$. It should be noted that the reentrant swelling occurs even in the case where the gap between $T_{N I}^{G}$ and $T_{N I}^{S}$ is rather narrow (for example, $2.8{ }^{\circ} \mathrm{C}$ for LCN-I/IV). At $T_{N I}^{S}$, nematic ordering of the surrounding solvent takes place, which does not cause discontinuous volume change but yields a kink of the $Q-T$ curve. In the totally nematic phase $\left(T<T_{N I}^{S}\right), Q$ is almost constant independently of $T$, and the magnitude is comparable to that in the totally isotropic phase $\left(T>T_{N I}^{G}\right)$. Figure 6 displays the optical micrographs for $\mathrm{LCN}-\mathrm{I} / \mathrm{IV}$ in the three characteristic temperature regions which clearly show the correlations of $Q$ with the phases $\mathbf{a}$

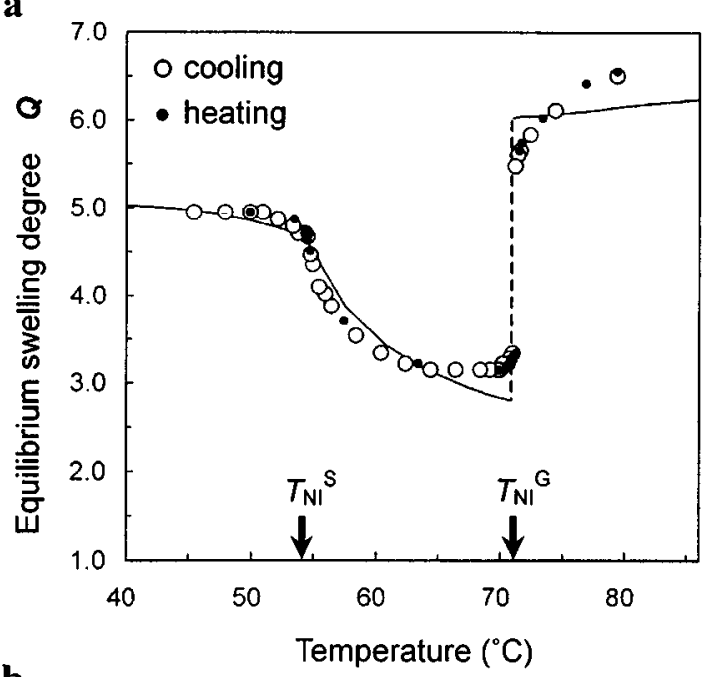

$\mathbf{b}$

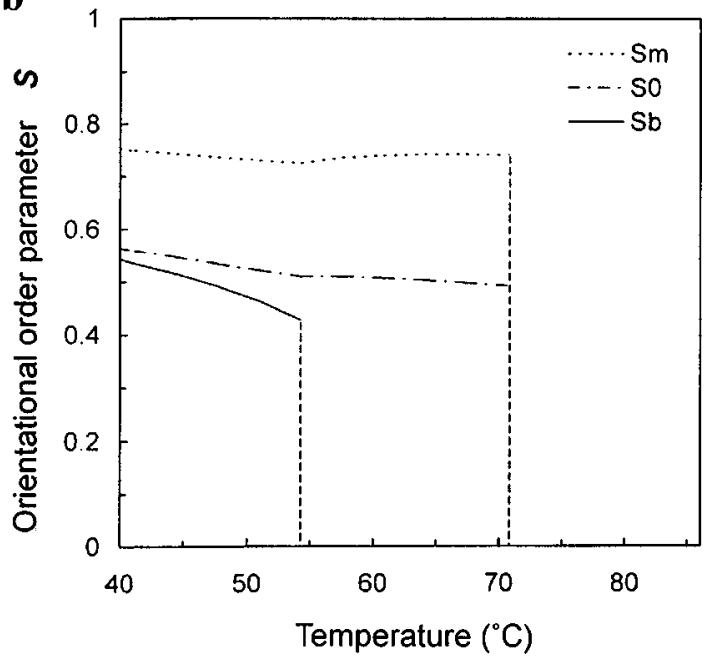

FIG. 4. (a) Equilibrium swelling degree $(Q)$ of LCN-II in the LC solvent III as a function of $T$. The nematic-isotropic transition temperature of the gel $\left(T_{N I}^{G}\right)$ and the surrounding pure LC solvent $\left(T_{N I}^{S}\right)$ are 71.0 and $54.2^{\circ} \mathrm{C}$, respectively. The solid line represent the theoretical swelling curve calculated with $n=25, n_{m}=5.1, p=0.15, n_{0}=2.5, \nu_{m m} / \nu_{00}=1.0, \quad \kappa=0.94$, $\nu_{00} / \chi=0.2$. (b) The orientational order parameters of the mesogen on network $\left(S_{m}\right)$, the nematic solvents inside and outside gel $\left(S_{0}\right.$ and $S_{b}$, respectively) as a function of $T$ calculated using the same parameter values as (a).

inside and outside the gel (also see Fig. 3 in Ref. 12 as the corresponding optical micrographs for LCN-I/III).

The $T$ dependence of $Q$ in heating process was also investigated for LCN-I/IV and LCN-II/III. As can be seen in Figs. 3 and 4, the significant effect of thermal history on the swelling and phase behavior was not observed except for a slight increase (within $0.5^{\circ} \mathrm{C}$ ) in $T_{N I}^{G}$ on heating.

It should be emphasized that the nematic network in the similar nematogen (LCN-II/IV) exhibits essentially the same swelling behavior as other systems composed of the nematic networks and the dissimilar nematogens, although the magnitude of the discontinuous volume change in LCN-II/IV is fairly smaller. This indicates that the swelling characteristics such as volume transition and reswelling are not due to the dissimilarity between mesogen on network and nematogen. 
$\mathbf{a}$

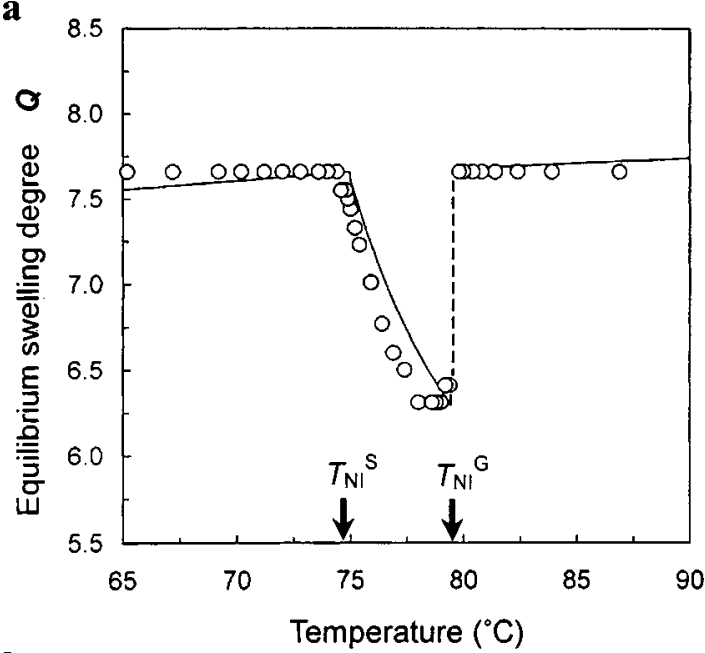

b

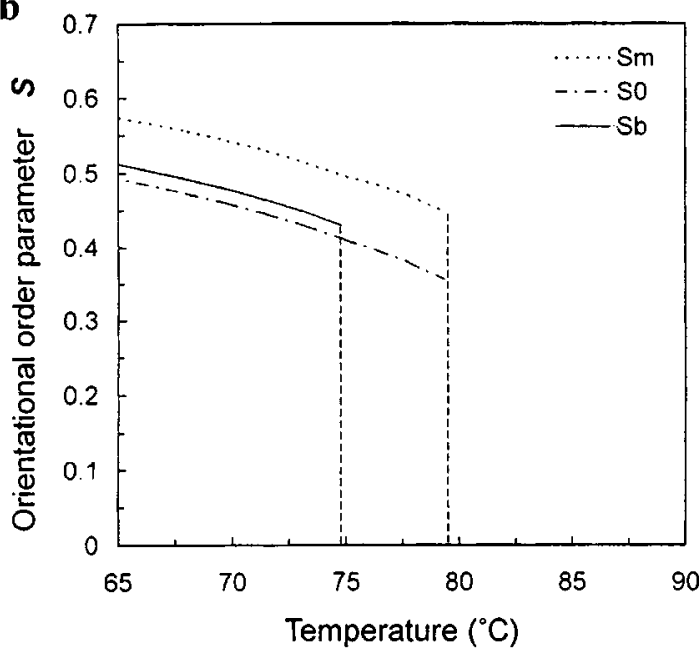

FIG. 5. (a) Equilibrium swelling degree $(Q)$ of LCN-II in the LC solvent IV as a function of $T$. The nematic-isotropic transition temperature of the gel $\left(T_{N I}^{G}\right)$ and the surrounding pure LC solvent $\left(T_{N I}^{S}\right)$ are 79.6 and $74.9^{\circ} \mathrm{C}$, respectively. The solid line represent the theoretical swelling curve calculated with $n=25, n_{m}=3.52, p=0.15, n_{0}=2.55, \nu_{m m} / \nu_{00}=1.0, \kappa=0.948$, $\nu_{00} / \chi=0.34$. (b) The orientational order parameters of the mesogen on network $\left(S_{m}\right)$, the nematic solvents inside and outside gel $\left(S_{0}\right.$ and $S_{b}$, respectively) as a function of $T$ calculated using the same parameter values as (a).

\section{DISCUSSION}

\section{A. Phase transition temperatures}

It is seen in Table I that $T_{N I}^{G}$ for the swollen LC gels is much reduced relative to $T_{N I}^{G}$ for the corresponding pure LC networks. This is qualitatively because the gel contains a large amount of the miscible nematic solvent whose $T_{N I}^{S}$ is rather lower than $T_{N I}^{G_{*}}$. We have found that the relation among $T_{N I}^{G}, T_{N I}^{G}$, and $T_{N I}^{S}$ is well expressed by the following simple additivity:

$$
T_{N I}^{G}=\left(1-\phi_{N I}\right) T_{N I}^{S}+\phi_{N I} T_{N I}^{G},
$$

where $\phi_{N I}$ is the volume fraction of network in the vicinity of $T_{N I}^{G}\left(T=T_{N I}^{G}+0.2^{\circ} \mathrm{C}\right)$ in the swollen phase. This equation is entirely phonomenological, but as can be seen in Table I, $T_{N I}^{G}$ calculated by Eq. (15) are fairly close to the data for all the systems. This good agreement is surprising, because $T_{N I}^{G}$ where the order parameter inside the gel jumps from zero to

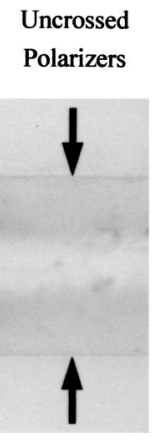

(a) $79.4^{\circ} \mathrm{C}$

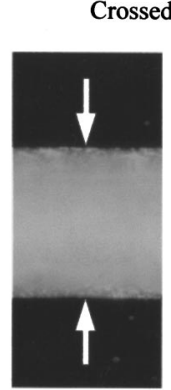

(b) $77.5^{\circ} \mathrm{C}$
Urayama et al.

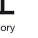


only alter the magnitudes of $Q$ and $T_{N I}^{G}$. The successful data fit is substantially attributed to the framework of the mean field theory. The mean field approach assuming monodomain LC structure in nematic phase may not be directly applicable to polydomain systems, but the good predictive capability demonstrated here suggests that the errors are fairly small for equilibrium swelling degree. The parameter values characterizing each nematic network or solvent were fixed in the data fitting for different systems: $n_{0}=2.5$ for the solvent III, $n_{0}$ $=2.55$ for the solvent IV, $n=120, n_{m}=3.3$, and $p=0.23$ for LCN-I, $n=25, p=0.15$ for LCN-II were consistently employed. The different values for $n_{m}$ of LCN-II, however, were required to achieve the good data fit in LCN-II/III and LCN-II/IV. Although the mesogen of LCN-II and the nematogen IV are similar, the fitted values of $n_{m}$ and $n_{0}$ are not identical. As mentioned before, the dangling mesogennetwork backbone interaction significantly influences the thermal stability of the nematic phase of the mesogen. For example, $T_{N I}^{G_{*}}$ for the dry LCN-II is much higher than $T_{N I}^{S}$ of the LC IV. The similar tendency is known for uncross-linked side-chain type LC polymers. ${ }^{34,35}$ The side group mesogennetwork backbone interaction is reflected in $n_{m}$ or $\nu_{m m}$ in the theory. The amounts of crosslinker truly introduced during synthesis are presumably lower than that given by the crosslinker concentration $(1 \mathrm{~mol} \%)$ in preparation, because the crosslinking efficiency is reduced by the presence of the mesogen. However, it should be noted that each LC network exhibited good reproducibility for $Q$ : The difference in $Q$ between the samples made separately was within $10 \%$. This is why the same value of $n$ was employed for each LC network in different solvents.

The $T$ dependence of each order parameter not only agrees with the phase observation, but also indicates that the swelling behavior is mainly governed by orientational order of each LC molecule. The equilibrium swelling in the totally isotropic phase $\left(T>T_{N I}^{G}\right.$ where there exists no orientational order) corresponds to the familiar case, namely, swelling of isotropic network in isotropic solvent. In this region, the $T$ dependence of $Q$ is mainly controlled by isotropic mixing interaction. The weak $T$ dependence of $Q$ in the totally isotropic phase stems from the weak $T$ dependence of $\chi(\sim 1 / T)$. At $T_{N I}^{G}$, the order parameters $S_{m}$ for mesogen on network and $S_{0}$ for the solvent inside the gel simultaneously jump from zero to finite values, which accords with the observation that the nematic network and the nematic solvent inside the gel form a single nematic phase. The theory demonstrates that the jump of $S_{m}$ and $S_{0}$, i.e., nematic ordering inside the gel, causes a discontinuous volume decrease (volume transition). In the region $T_{N I}^{S}<T<T_{N I}^{G}$, the nematic order inside the gel $\left(S_{m}\right.$ and $\left.S_{0}\right)$ increases with decreasing in $T$ in a similar way as $S$ of general LC in the nematic phase does, ${ }^{27,36}$ whereas the surrounding solvent remains isotropic $\left(S_{b}=0\right)$. This indicates that the reswelling, i.e., an uptake of the surrounding isotropic LC solvent by the nematic network, is thermodynamically induced by an increase in orientational order inside the gel. The theoretical swelling curve has an inflection at $T_{N I}^{S}$ as a result of the jump of $S_{b}$ from zero to a finite value. Nematic ordering of the pure solvent outside the gel does not cause an instability in the chemical potential of the gel, and consequently, the gel volume changes continuously at $T_{N I}^{S}$. The order parameters inside the gel still increase with decreasing in $T$ in the totally nematic phase $\left(T<T_{N I}^{S}\right)$, which does not yield further swelling in contrast to the region $T_{N I}^{S}<T<T_{N I}^{G}$. When the nematic orders inside and outside the gel are comparable, the growth of the nematic order inside the gel does not significantly alter the degree of equilibrium swelling. It should be emphasized again that a further swelling induced by an increase in nematic order inside the gel occurs when there exists no nematic order in the surrounding solvent $\left(S_{b}=0\right)$.

In the mean field theory, the difference between $T_{N I}^{G}$ and $T_{N I}^{S}$ mainly depends on $n_{m} / n_{0}, \nu_{m m} / \nu_{00}$ and $\kappa$. As each of these values increases, the ratio $T_{N I}^{G} / T_{N I}^{S}$ becomes higher. High axial ratio of mesogen $\left(n_{m}\right)$ as well as large nematic interaction between mesogens on network $\left(\nu_{m m}\right)$ stabilizes the nematic phase of the gel, which increases $T_{N I}^{G}$. An increase in $\kappa$ representing cross-interaction between mesogen on network and nematic solvent also stabilizes the nematic phase of the gel comprising the mixture of the mesogen and the solvent. For all the systems, the fitted values of $\kappa$ are less than unity. This agrees with the results $(\kappa<1)$ reported for general LC mixtures: ${ }^{23,28,29}$ Unless there exists strong crossinteraction between the two dissimilar nematogens, $\kappa$ is less than unity.

In the case of nonionic isotropic gels such as $N$-isopropylacrylamide gel, a specific dependence of $\chi$ (polymer-solvent mixing interaction) on temperature and/or polymer concentration is an important key to the volume transition phenomena. ${ }^{11}$ Meanwhile, in the case of nematic gel, none of the isotropic mixing interactions between mesogen, spacer and solvent does not play an important role in the volume transition and reentrant swelling, although they influence the magnitude of $Q$. The characteristic swelling behavior is observed even in the system with $\chi_{m 0} \approx 0$ (LCNII/IV; a nematic network in a similar nematogen); even if $\chi\left(=\chi_{m s}=\chi_{s 0}\right)=0$ is assumed, the theory predicts that nematic gel exhibits the volume transition as well as reentrant swelling. Thus nematic order is a governing force for the characteristic swelling behavior of nematic gel.

As mentioned before, the mean-field theory is originally derived on the basis of main-chain LC network. The dangling mesogen-network backbone interaction in side-chain LC polymers is not explicitly introduced in the mean field theory. This interaction appears mainly in $T_{N I}^{G}$, and as a result, it is reflected in $n_{m}$ and $\nu_{m m}$ in the data fit as in the case of the mixtures of side-chain LC polymers and nematic solvents. $^{23,24}$ The backbone of side-chain LC network is totally composed of nonmesomorphic components, which may influence on the solubility. This is important in isotropic solvent system, but minor in nematic solvent: As described before, even if we drop the isotropic mixing interaction parameters $(\chi)$ in the free energy, the theory predicts essentially the swelling features, i.e., volume transition and reswelling behavior. The use of the conventional $\chi$ parameter is sufficient to reflect the effect of totally nonmesomorphic backbone on solubility. Thus, as in the case of the mixtures of side-chain LC polymers and nematic solvent, ${ }^{23,24}$ the mean field theory is at least qualitatively successful in describing the swelling 
and phase behavior of the side-chain LC gels in nematic solvent. The comparison of the swelling and phase behavior between main- and side-chain LC gels comprising identical mesogens will provide us important information about this issue. This is a subject in our future study.

\section{CONCLUSIONS}

The equilibrium swelling behavior of nematic network in miscible nematic solvent is mainly governed by nematic (orientational) orders of the LC molecules inside and outside the gel. The degree of equilibrium swelling $(Q)$ is almost independent of $T$ in the totally isotropic phase $\left(T>T_{N I}^{G}\right)$ where there exists no orientational order in the system as well as in the totally nematic phase $\left(T<T_{N I}^{S}\right)$ where the nematic orders inside and outside the gel are comparable. Meanwhile, $Q$ is strongly $T$ dependent in the region $T_{N I}^{S}<T$ $<T_{N I}^{G}$ where there exists a finite nematic order inside the gel whereas nematic order outside the gel is absent. When the isotropic swollen gel is cooled down to $T_{N I}^{G}$, the nematic ordering inside the gel takes place, which simultaneously causes a discontinuous reduction in gel volume (nematic ordering-induced volume transition); that is, the isotropic swollen gel is discontinuously collapsed into the nematic gel. When the system is further cooled, the shrunken nematic gel swells again with descending in $T$ as a result of an increase in nematic order inside the gel. Due to this reswelling, $Q$ of the nematic gel at $T \approx T_{N I}^{S}$ becomes comparable to that in the totally isotropic phase. At $T_{N I}^{S}$, the nematic ordering of the solvent outside the gel occurs, which yields a kink of the $Q-T$ curves without discontinuity. No significant effect of thermal hysteresis on the swelling and phase behavior is observed. The correlation between swelling and phase characteristics does not depend on the details of chemical structures of mesogen on network and nematic solvent. A nematic network in a similar nematogen also undergoes a nematic ordering induced-volume transition as well as a reswelling in the range in $T_{N I}^{S}<T<T_{N I}^{G}$. The experimental $Q-T$ curves and the phase behavior observed are successfully described by a mean field theory where nematic ordering effect is considered on the basis of the Maier-Saupe concept. The theory also demonstrates that nematic order of LC molecule is a governing force for swelling of nematic gel: The nematicisotropic transition inside gel drives a volume transition; an increase in nematic order inside gel thermodynamically induces a further swelling of nematic gel surrounded by isotropic LC solvent.

\section{ACKNOWLEDGMENT}

The numerical calculation was performed with the approval of Academic Center for Computing and Media Studies, Kyoto University.

${ }^{1}$ K. Dusek and D. Patterson, J. Polym. Sci., Part A-2 6, 1209 (1968).

${ }^{2}$ T. Tanaka, Phys. Rev. Lett. 40, 820 (1978).

${ }^{3}$ T. Amiya and T. Tanaka, J. Chem. Phys. 86, 2375 (1987).

${ }^{4}$ M. Annaka and T. Tanaka, Nature (London) 355, 430 (1992).

${ }^{5}$ S. Kang and Y. H. Bae, Macromolecules 34, 8173 (2001).

${ }^{6}$ Y. Hirokawa, T. Tanaka, and E. S. Matsuo, J. Chem. Phys. 81, 6379 (1984).

${ }^{7}$ F. Ilmain, T. Tanaka, and E. Kokufuta, Nature (London) 349, 400 (1991).

${ }^{8}$ M. Tokita and T. Tanaka, Science 253, 1121 (1991).

${ }^{9}$ S. Seelenmeyer, I. Deike, S. Rosenfeldt, C. Norhausen, N. Dingenouts, M. Ballauff, T. Narayanan, and P. Lindner, J. Chem. Phys. 114, 10471 (2001).

${ }^{10}$ X. Z. Zhang, Langmuir 17, 6094 (2001).

${ }^{11}$ M. Shibayama and T. Tanaka, Adv. Polym. Sci. 109, 1 (1993).

${ }^{12}$ K. Urayama, Y. Okuno, T. Kawamura, and S. Kohjiya, Macromolecules 35, 4567 (2002).

${ }^{13}$ T. Okano, Adv. Polym. Sci. 110, 180 (1993).

${ }^{14}$ R. Yoshida, K. Uchida, Y. Kaneko, K. Sakai, A. Kikuchi, Y. Sakurai, and T. Okano, Nature (London) 374, 240 (1995).

${ }^{15} \mathrm{C}$. Alvarez-Lorenzo and A. Concheiro, J. Controlled Release 80, 247 (2002).

${ }^{16}$ R. Zentel, Liq. Cryst. 1, 589 (1986).

${ }^{17}$ R. Kishi, Y. Suzuki, H. Ichijo, and O. Hirasa, Chem. Lett. 1994, 2257.

${ }^{18}$ R. Kishi, Y. Suzuki, H. Ichijo, and O. Hirasa, Mol. Cryst. Liq. Cryst. 295, 113 (1997).

${ }^{19}$ X. J. Wang and M. Warner, Macromol. Theory Simul. 6, 37 (1997).

${ }^{20}$ A. Matsuyama and T. Kato, Phys. Rev. E 64, 010701 (2001).

${ }^{21}$ A. Matsuyama and T. Kato, J. Chem. Phys. 116, 8175 (2002).

${ }^{22}$ M. Warner, K. P. Gelling, and T. A. Vilgis, J. Chem. Phys. 88, 4008 (1988).

${ }^{23}$ H.-W. Chiu and T. Kyu, J. Chem. Phys. 103, 7471 (1995).

${ }^{24}$ H.-W. Chiu, Z. L. Zhou, T. Kyu, G. L. Cada, and L. C. Chien, Macromolecules 29, 1051 (1996).

${ }^{25}$ P. J. Flory, Principles of Polymer Chemistry (Cornell University Press, Ithaca, 1953).

${ }^{26}$ W. Maier and A. Z. Saupe, Naturforsh, A. 14a, 882 (1959).

${ }^{27}$ P. G. de Gennes and J. Prost, The Physics of Liquid Crystals, 2nd ed. (Oxford University Press, New York, 1993).

${ }^{28}$ F. Brochard, J. Jouffroy, and P. Levinson, J. Phys. (France) 45, 1125 (1984).

${ }^{29}$ F. Benmouna, U. Maschke, X. Coqueret, and M. Benmouna, Polym. Int. 50, 469 (2001).

${ }^{30}$ F. Brochard, J. Phys. (France) 40, 1049 (1979).

${ }^{31}$ A. Matsuyama and T. Kato, J. Chem. Phys. 114, 3817 (2001).

${ }^{32}$ F. Benmouna, J. Ruhe, and D. Johannsmann, Liq. Cryst. 26, 1655 (1999).

${ }^{33}$ D. Nwabunma and T. Kyu, Macromolecules 32, 664 (1999).

${ }^{34}$ N. A. Plate and V. P. Shivaev, Comb-shaped Polymers and Liquid Crystals (Plenum, New York, 1987).

${ }^{35}$ D. J. Simmonds, in Liquid Crystalline Polymers: From Structures to Applications, edited by A. A. Collyer (Elsevier Applied Science, London, 1992).

${ }^{36} \mathrm{~S}$. Chandrasekhar, Liquid Crystals, 2nd ed. (Cambridge University Press, Cambridge, 1992). 